\title{
Wie setzen sich angehende Lehrkräfte mit pädagogischen Situationen auseinander? Eine Analyse von Argumentationsstrukturen und genutzten Informationen
}

\author{
Kati Trempler $(\mathbb{D} \cdot$ Ulrike Hartmann
}

Eingegangen: 5. Juni 2019 / Überarbeitet: 29. Mai 2020 / Angenommen: 17. September 2020 / Online publiziert: 30 . Oktober 2020

(C) Der/die Autor(en) 2020

Zusammenfassung Die kritische Auseinandersetzung mit pädagogischen Situationen ist eine bildungsrelevante Fähigkeit, die Lehrkräfte ihren Schülerinnen und Schülern vermitteln und die sie selbst im Sinne einer evidenzorientierten Praxis nutzen sollten, um ihre eigenen Entscheidungen in Bezug auf ihr professionelles Handeln zu begründen. Kritisches Denken zeigt sich in der Fähigkeit zu argumentieren und in der Art der genutzten Informationen (z. B. wissenschaftliches Wissen, Erfahrungen). Die Wahrnehmung, Darstellung und argumentative Abwägung verschiedener Positionen und Informationen weist insbesondere auf kritische Denkprozesse hin. In der vorliegenden Studie stellen wir daher die Fragen, (1) ob Lehramtsstudierende bei der schriftlichen und individuellen Auseinandersetzung mit pädagogischen Situationen argumentieren und wenn ja, welche Struktur diese Argumentationen aufweisen, (2) welche Informationen sie bei der Auseinandersetzung mit pädagogischen Situationen nutzen und (3) in welchem Zusammenhang genutzte Informationen mit der Argumentationsstruktur stehen. Wir analysierten 87 schriftliche Auseinandersetzungen von Studierenden im Master of Education, in denen sie eine schulische Situation auswählen, beschreiben und erklären sollten, und werteten diese Einträge mit einem qualitativ-quantifizierenden Vorgehen aus. Es wurden die Argumentationsstruktur (non-argumentation, einseitige Argumentation, zweiseitige Argumentation) sowie die Informationsintegration (intern, extern, intern und extern) mit einem eigens entwickelten Kodierschema untersucht. Die Ergebnisse zeigen,

\footnotetext{
Dr. K. Trempler $(\bowtie)$

Arbeitsbereich: Lehr-, Lern- und Unterrichtsforschung, Institut für Bildungsforschung der Bergischen Universität Wuppertal, Gaußstraße 20, 42119 Wuppertal, Deutschland

E-Mail: trempler@uni-wuppertal.de

Dr. U. Hartmann

Leibniz-Institut für Bildungsforschung (DIPF), Rostocker Straße 6, 60323 Frankfurt am Main, Deutschland

E-Mail: u.hartmann@dipf.de
} 
dass Lehramtsstudierende vor allem einseitig argumentieren; ein Viertel der Studierenden integriert verschiedene Positionen im Sinne einer zweiseitigen Argumentation. Zudem nutzen Studierende bei der schriftlichen Auseinandersetzung größtenteils externe Informationen (z. B. Lehrbücher). Die Fähigkeit zweiseitig zu argumentieren steht darüber hinaus in Zusammenhang mit der Informationsintegration. Die Ergebnisse werden kritisch in Hinblick auf die Konsequenzen für die Lehrerbildung diskutiert.

Schlüsselwörter Kritsiches Denken · Argumentation · Informationsintegration · Lehrerbildung · Pädagogische Situationen · Evidenzorientierte Praxis

\title{
How do prospective teachers deal with pedagogical situations? An analysis of argumentation structures and used information
}

\begin{abstract}
Critical thinking is a relevant educational skill that teachers should impart to their students and use themselves in an evidence-oriented practice to justify their own decisions regarding their professional actions. Critical thinking comprises the ability to argue and integrate different information (i.e. scientific knowledge to experiences). The perception, presentation and weighing of different positions and information points in particular to critical thinking-processes. In this study, we therefore ask the questions (1) whether teachers argue in their written examination of pedagogical situations and, if so, what structure these arguments have, (2) which information they use in dealing with pedagogical situations and (3) if the information used are related to the structure of arguments. We analyzed 87 written disputes of prospective teachers in which they should select, describe and explain a pedagogical situation they encountered. We evaluated these entries with a qualitative-quantifying approach. Argumentation structure (non-argumentation, one-sided argumentation, two-sided argumentation) as well as integration of information (internal, external, internal \& external) were examined with a newly developed coding scheme. The results show that prospective teachers argue mainly on the basis of one position; a quarter of students integrate different positions (two-sided argument with arguments and counter-arguments). In addition, students largely use external information (e.g. textbooks) within their written dispute and the ability to argue on two sides is related to the integration of information (combination of intern and extern information). The results will be critically discussed with regard to the consequences for teacher education.
\end{abstract}

Keywords Argumentation - Critical thinking - Evidence-oriented practice · Integration of infomation · Pedagogical situation · Teacher education

\section{Einleitung}

Wir leben in einer Wissensgesellschaft und sind oft von vielen Informationen umgeben, die für private wie auch berufliche Angelegenheiten relevant sind. 
In der öffentlichen Diskussion werden bspw. Inhalte aus den Bereichen Gesundheit, Umwelt oder Bildung kontrovers diskutiert. Dabei zeigt sich häufig, dass aufgrund verschiedener Geltungsbehauptungen nicht die eine Information - seien es Aussagen anderer Personen oder Beiträge in Medien - genügt, um eine hinreichende Informationsbasis aufzubauen und eine fundierte Entscheidung zu treffen (Bromme und Kienhues 2014; Sinatra 2016). Stattdessen ist eine sorgsame Betrachtung und Abwägung verschiedener Informationen unabdingbar (Fischer et al. 2014; Asterhan und Schwarz 2016).

Diese Herausforderung kann ebenso auf die pädagogische Praxis übertragen werden: Lehrkräfte müssen täglich mit komplexen, unsicheren Unterrichtssituationen umgehen und die vor dem Hintergrund des jeweiligen Lernziels beste, verantwortbare Entscheidung anhand bestehender Informationen treffen (Cochran-Smith und Lytle 1999; Berliner 2002; Tenorth 2006). Vor allem die Nutzung bildungswissenschaftlicher Informationen für Entscheidungen in pädagogischen Situationen wird in den letzten Jahren vermehrt im Zuge der Evidenzorientierung im Lehrerberuf diskutiert (Hartmann et al. 2016; Bauer et al. 2017).

Die evidenzorientierte bzw. informationsbasierte Auseinandersetzung mit pädagogischen Situationen erfordert auf Seiten der Lehrkräfte komplexe Fähigkeiten, die u. a. die Wahrnehmung und das Verstehen einer Situation, die Informationssuche und -bewertung, die argumentative und reflektierte Auseinandersetzung sowie das Ableiten alternativer Handlungen umfassen (Leinhardt und Greeno 1986; Trempler et al. 2015; Bauer et al. 2017). Das Ausführen dieser Fähigkeiten hat positive Wirkungen für Lehrkräfte zur Folge, wie bspw. erhöhtes Wohlbefinden (Wubbels und Korthagen 1990; Mattern und Bauer 2014) sowie eine bessere Unterrichtswahrnehmung (Mertens und Gräsel 2018). Zudem können Fähigkeiten der kritischen Auseinandersetzung von Lehrkräften direkt z. B. in Form eines Modelling an die Lernenden übertragen werden (Muis et al. 2006), was wiederum aus der Sicht nationaler wie internationaler Bildungsstandards als wünschenswert erachtet wird (Common Core State Standards 2010; KMK 2012). Lernende sollten demnach in der Lage sein, selbständig notwendige Informationen zu suchen und zu bewerten, eine Wissensbasis zu entwickeln, Perspektiven zu erweitern und überzeugend zu argumentieren, um im privaten und im öffentlichen Leben Verantwortung zu übernehmen. Darin ist der Auftrag an das Bildungssystem und die darin agierenden Lehrkräfte gut erkennbar: Sie sollten dazu befähigt sein, flexibel, reflektiert und kritisch mit herausfordernden pädagogischen Situationen umzugehen, deren Passung an die Bedürfnisse der Lernenden zu prüfen sowie Erkenntnisse über den Erwerb von Wissen und Fähigkeiten zur Gestaltung erfolgreicher Lernprozesse in den Unterricht zu integrieren (KMK 2014). Die Entwicklung dieser Fähigkeiten ist daher nicht nur als Ziel schulischer, sondern auch als Ziel universitärer Bildung zu begreifen (Fischer et al. 2014).

Aus diesem Grund beschäftigen wir uns im vorliegenden Beitrag mit der Fähigkeit angehender Lehrkräfte sich kritisch mit pädagogischen Situationen auseinanderzusetzen. Ausgehend vom Ansatz des kritischen Denkens und der Argumentationsfähigkeit gehen wir mit unserer qualitativen Studie zum einen der Fragestellung nach, ob angehende Lehrkräfte sich argumentativ mit pädagogischen Situationen auseinandersetzen. Zum anderen fragen wir, welche Informationen sie für diese Auseinandersetzung nutzen. 
Die Besonderheit des vorliegenden Projekts liegt darin, dass die Studierenden sich mit realen Situationen beschäftigen, die sie während einer verlängerten Praxisphase selbst aussuchen. Durch die Situierung im naturalistischen Kontext können spontane Auseinandersetzungen mit Praxissituationen herausgearbeitet werden, die von angehenden Lehrkräften als relevant für ihre berufliche Tätigkeit eingeschätzt werden. Im Zuge der Debatte um evidenzorientiertes Handeln, das sowohl den Rückbezug auf eigene berufliche Erfahrungen als auch auf die beste verfügbare wissenschaftliche Evidenz erfordert, beschränken wir uns nicht auf die Nutzung wissenschaftlicher Informationen, sondern erweitern diesen Blick auf alle verfügbaren Informationen (theoretisches Wissen, empirisches Wissen, Erfahrungen, Beobachtungen) (Kuhn 1991; Chinn et al. 2011).

\section{Theoretischer Hintergrund}

\subsection{Kognitive Komponenten bei der Auseinandersetzung mit pädagogischen Situationen}

Bei der Auseinandersetzung mit komplexen pädagogischen Situationen sind spezifische Fähigkeiten von Bedeutung, die auf einer allgemeinen theoretischen Ebene als Reflexion bezeichnet werden können. In den Worten Alexanders (2017, S. 308) beschreibt Reflexion ,....) the deliberation, pondering, or rumination over ideas, circumstances, or experiences yet to be enacted, as well as those presently unfolding or already passed“. Sie kann demnach während einer Situation stattfinden (z. B. reflection-in-action, Schön 1983) als auch in Vor- und Nachbereitung einer Situation genutzt werden (Schön 1983; Korthagen und Wubbels 2008; Farrell 2012). Start und Endpunkt eines Reflexionsprozesses stellen professionelle Handlungen dar, die durch kritische informationsbasierte Reflexionsprozesse weiterentwickelt werden. So beschreiben Korthagen und Wubbels (2008; ALACT-Modell) diesen Prozess in fünf Schritten, bestehend aus einer Handlung, der Betrachtung der Handlung, der Bewusstmachung kritischer Aspekte, der Generierung alternativer Handlungsoptionen und der Anwendung letzterer.

Lunn Brownlee et al. (2017) zeigen in einem Prozessmodell, wie die genannten Fähigkeiten im pädagogischen Kontext gedacht werden können: Wenn Lehrkräfte ihren eigenen Unterricht entsprechend bestimmter epistemischer Ziele (z. B. Verstehen, Wissensaneignung usw.) planen und dieser in einer anderen Form als geplant verläuft, kann dies als Problem oder Diskrepanz wahrgenommen werden. Diese Diskrepanz kann anschließend durch einen internen Dialog (S. 248) oder die reflective conversation with a situation (Schön 1983, S. 163) und anhand verschiedener Informationen erklärt werden. Daraufhin werden Erklärungsmodelle identifiziert, die das gewünschte Ergebnis eventuell beeinflussen, alternative Handlungsoptionen entsprechend ihrer hypothetischen Wirkung eruiert und abschließend eine Schlussfolgerung für das künftige Handeln abgeleitet. Der „kritische“ Teil dieses Denkprozesses besteht dabei in der Prüfung des angewandten Wissens auf seine Gültigkeit für die jeweilige Situation (Hofer 2016). Reflexion kann dementsprechend als Instrument zur Verbindung von Theorie und Praxis verstanden werden (Korthagen 2010). 
Bisherige Untersuchungen im Bereich der Theorie-Praxis-Verzahnung zeichnen jedoch ein eher pessimistisches Bild und weisen darauf hin, dass Lehrkräfte Schwierigkeiten haben ihr im universitären Kontext erworbenes Wissen sowie Fähigkeiten auf den schulischen Kontext zu übertragen und stattdessen Gefahr laufen, auf bereits bestehende Routinen zurückzugreifen (Korthagen 2010). Diese Befunde werden durch aktuelle Studien untermauert, die zum einen zeigen, dass vor allem Novizinnen und Novizen durch komplexe pädagogische Situationen stark beansprucht und überfordert (Kim und Klassen 2018) sowie deren Fähigkeiten zur Wahrnehmung, Erklärung und Interpretation problematischer pädagogischer Situationen stark eingeschränkt sind (Stürmer et al. 2013; Wolff et al. 2016). Diese Befunde könnten dadurch erklärt werden, dass Studierende bereits im Lehramtsstudium Schwierigkeiten bei der Reflexion pädagogischer Situationen aufweisen: Kiemer und Kollar (2018) beschäftigen sich in einer aktuellen Studie mit der evidenzbasierten Reflexion angehender Lehrkräfte aus der Perspektive der Script-Theorie (Fischer et al. 2013). Die Ergebnisse weisen darauf hin, dass angehende Lehrkräfte in Bezug auf die kognitiven Prozesse während der Reflexion unvollständige Scripts in nicht ausreichender Qualität aufweisen. Geringe Fähigkeitswerte zeigen sich in Bereichen informationsbasierter Erklärungen von Problemsituationen und der Ableitung von Zielen und Handlungsoptionen. Ähnliche Ergebnisse berichten Nückles und Schuba (2019) in ihrer aktuellen Studie zur Reflexion und Planung von Unterricht auf der Basis wissenschaftlichen Wissens durch angehende Lehrkräfte anhand von Lerntagebüchern. Die Ergebnisse weisen darauf hin, dass angehende Lehrkräfte für die jeweilige Situation relevante wissenschaftliche Informationen identifizieren und auch allgemeine Handlungsoptionen aus diesen Texten filtern können, allerdings keine informationsbasierten Handlungsalternativen für die jeweilige Situation generieren können.

Es kann angenommen werden, dass die geschilderten Reflexionsfähigkeiten auch durch intensive Berufserfahrung von Lehrkräften nicht automatisch (re)aktiviert werden (Lachner et al. 2016). Aus diesem Grund scheint es plausibel, sich bereits in einer früheren Phase der Lehrkräfteausbildung mit Fähigkeiten der informationsbasierten Reflexion auseinanderzusetzen. Da Lehramtsstudierende in der Regel noch nicht über umfangreiche praktische Erfahrungen verfügen und somit noch keine hinreichende Basis professionellen Wissens (bestehend aus Erfahrungen und wissenschaftlichem Wissen) entwickeln konnten, gehen wir davon aus, dass eine unmittelbare Reflexion in der jeweiligen Situation, wie Schön (1983; reflection-inaction) sie vorstellt, für Studierende nicht realistisch ist. Vielmehr wird die Reflexion angehender Lehrkräfte im vorliegenden Beitrag als prä- oder postaktionale Phase begriffen (reflection-on-action, reflection-for-action), in der Studierende ihre praktischen Erfahrungen forschend nutzen, diese anhand verschiedener Informationsbestände interpretieren und Schussfolgerungen für künftige Handlungen ziehen (Cochran-Smith und Lytle 1999). Bisher sind diese Auseinandersetzungsprozesse jedoch noch wenig spezifiziert. Wir gehen davon aus, dass die systematische, informationsbasierte Auseinandersetzung mit pädagogischen Situationen als spezifische Fähigkeit des kritischen Denkens beschrieben werden kann. Kritisches Denken wird als höhere Form des Denkens verstanden (Zohar und Nemet 2002; Osborne und Patterson 2011; Lunn Brownlee et al. 2017), bei der Personen sich aktiv, selbstreguliert 
und gewissenhaft mit ihren Überzeugungen und vorliegenden Informationen auseinandersetzen, daraus neue Wissensstrukturen entwickeln und begründete Entscheidungen ableiten (Cochran-Smith und Lytle 1999; Fisher 2011). Entsprechend klassischer Zwei-Prozess-Modelle der Informationsverarbeitung kann kritisches Denken als systematische Verarbeitung bezeichnet werden, bei der neues Wissen aufgenommen und in bestehende Wissensstrukturen integriert wird (Chaiken 1980). Zu den Teilfähigkeiten kritischen Denkens zählen u. a. die Identifikation und Interpretation von Problemen in einer Situation, die Suche valider Informationen für die Erklärung der Situation, das Kontrollieren von Einflussfaktoren, die Analyse und Bildung von Argumenten und Gegenargumenten sowie die Ableitung von Schlussfolgerungen und Handlungsalternativen (Leitão 2000; Abrami et al. 2015).

Zusammenfassend umfassen kritische Auseinandersetzungsprozesse mit pädagogischen Situationen zum einen die Fähigkeit des Argumentierens und zum anderen die Nutzung von Informationen in Verbindung mit Argumentationen. Diese Teilfähigkeiten kritischen Denkens stehen im Fokus des vorliegenden Beitrages.

\subsubsection{Argumentation}

Die Analyse von Argumentationen kann ein Weg sein, Denkprozesse angehender Lehrkräfte bei der Auseinandersetzung mit pädagogischen Situationen zu visualisieren. Insbesondere die Konstruktion von Argumenten und Gegenargumenten und mit ihnen die Auswahl und Bewertung von Informationen, die die Argumente stützen, werden dabei als wesentliche Faktoren des kritischen Denkens betrachtet (Newell et al. 2011). Insbesondere die Forschung zur Entwicklung der Fähigkeit des Argumentierens beschäftigt sich mit der Analyse von Prozessen des kritischen Denkens anhand von Argumentationen (Clark und Linn 2013; Duncan und Chinn 2016; Kuhn 1991; Mercier und Sperber 2011; Sinatra 2016).

Argumentationen stellen eine Form der dialektischen Auseinandersetzung dar, die zwischen Individuen, aber auch allein in Form eines internen Dialogs stattfinden kann (Nussbaum 2008). Bei dieser Auseinandersetzung reagieren Personen auf Herausforderungen oder Fragen, die durch einen so genannten Interlocutor - also einen Gesprächspartner - gestellt werden (Kuhn 2015). Die Argumentation kann zudem schriftlich oder mündlich erfolgen, wobei gerade in individuellen, schriftlichen Auseinandersetzungen die Bildung von Gegenargumenten hervorzuheben ist, da aufgrund des fehlenden Gesprächspartners eine zweite Position selbst entwickelt werden muss (Iordanou 2010).

\subsubsection{Struktur und Qualität von Argumentationen}

Entsprechend klassischer Definitionen besteht eine vollständige Argumentation aus einer oder mehreren Behauptungen, die durch Argumente und Gegenargumente gerechtfertigt und aus denen Konklusionen abgeleitet werden (Zohar und Nemet 2002; Toulmin 2003; Duncan und Chinn 2016).

Eine Behauptung stellt eine einfache, ungerechtfertigte Aussage dar, die erst durch eine Rechtfertigung in Form einer Information zum Argument wird (Kuhn 1991). Behauptungen und Argumente unterscheiden sich demnach durch das Vorhandensein 
einer Information, die als Rechtfertigung dient (Toulmin 2003; Zohar und Nemet 2002). Konklusionen können entweder auf der Grundlage von informationsbasierten Argumenten entwickelt werden (reasoning, Moshman und Tarricone 2016) - wobei epistemische Ziele verfolgt werden, die den Aufbau und die Integration neuer Informationen, das Verstehen, die Bildung gerechtfertigter Überzeugungen und das Erlangen von Fähigkeiten umfassen (Chinn et al. 2011; Iordanou et al. 2016) - oder auf der Basis vorher bestehender Repräsentationen (inference; Mercier und Sperber 2011), bei denen kein Bezug zu Argumenten und neuen Informationen besteht.

Diese Definition stellt den Minimalkonsens der vielfältigen Definitionen zu Argumentationsstrukturen dar. Zudem werden zwei Formen der Argumentation unterschieden: Bei einer zweiseitigen Argumentation werden bewusst verschiedene Positionen (Argumente und Gegenargumente) aus bereits vorhandenen Informationen und neuen Informationen von außen gegenübergestellt (Mercier und Sperber 2011; Iordanou et al. 2016). Mit Bezug zum Lehrerberuf könnte das bedeuten, dass Erfahrungswissen (bspw. zum Verhalten von Lernenden) durch Informationen von außen (bspw. bildungswissenschaftliche Befunde) ergänzt wird und in Schlussfolgerungen mündet, die zu alternativen Handlungen führen. Zweiseitige Argumentation führt im besten Fall zur Bildung neuer Wissensbestände und wird daher als Grundlage für das Ändern von Denkweisen und Überzeugungen angesehen (Fisher 2011; Iordanou et al. 2016). Diese Form der Argumentation wird als Voraussetzung dafür angesehen, schnell und intelligent auf konstanten Wandel, Probleme und Herausforderungen zu reagieren sowie Innovationen voranzubringen (Nussbaum 2008; Crowell und Kuhn 2014). All dies sind Aspekte, die für das spätere Unterrichten essenziell erscheinen.

Als einseitige Argumentation werden dementgegen Argumentationsstrukturen bezeichnet, die keine widersprüchlichen Informationen (Gegenargumente) beinhalten und auf der Basis bereits bestehender Repräsentationen gebildet werden (Mercier und Sperber 2011). Sie zielen in der Regel nicht auf die Erlangung epistemischer Ziele ab, sondern auf die Ableitung von Schlussfolgerungen, die leicht zu rechtfertigen sind, und auf die Bekräftigung der eigenen Meinung (Hart et al. 2009; Walton und Macagno 2015). So konnten Hart et al. (2009) in einer Metanalyse zeigen, dass Personen dazu tendieren, externe Informationen zu wählen, die ihren eigenen Vorannahmen entsprechen, was zur Folge hat, dass sie kaum neue Informationen für Entscheidungen nutzen (Buehl et al. 2001; Maier et al. 2014).

Diverse Studien zeigen, dass die zweiseitige Argumentation und insbesondere die Ableitung und Rechtfertigung von Gegenargumenten eine besondere Herausforderung darstellen und Personen daher häufig dazu tendieren schon vorhandene Annahmen ohne Beachtung alternativer Positionen zu rechtfertigen (Hart et al. 2009; Asterhan und Schwarz 2016).

Für den Bildungsbereich liegen bereits verschiedene Befunde zur Argumentationsfähigkeit vor: Sie zeigen, dass nur wenige Kinder und Jugendliche als skilled arguers bezeichnet werden können, und dass die Fähigkeit zur Bildung zweiseitiger Argumentationen vor allem durch spezielle Argumentationstrainings gefördert werden kann (Iordanou 2010; Crowell und Kuhn 2014). Für die Seite der Lehrkräfte existieren bereits vereinzelte Studien, die auf eine verminderte Argumentations- und Reflexionsfähigkeit angehender Lehrkräfte hinweisen: So zeigen qualitative Studien, bei denen Lehrkräfte pädagogische Szenarien anhand von Laut-Denk-Protokol- 
len bearbeiten, dass Novizinnen und Novizen zwar in der Lage sind, Probleme in Situationen wahrzunehmen (wenn auch auf einem oberflächlicheren Niveau im Vergleich zu Expertinnen und Experten), aber Schwierigkeiten haben, die Situationen zu analysieren, daraus Schlussfolgerungen abzuleiten und Vorhersagen zu treffen. Die Autoren fassen zusammen, dass Novizinnen und Novizen schlechtere Argumentations- und Reflexionsfähigkeiten aufweisen - eventuell erklärbar durch eine höhere kognitive Auslastung (Kim und Klassen 2018, S. 223). Alle Gruppen weisen zudem eine Tendenz zu einseitigen Argumentationen auf, da sie ausschließlich eigene Erfahrungen nutzen. Inwieweit diese Aussagen auch auf die Auseinandersetzung mit realen Situationen zutreffen, kann die Studie nicht beantworten. Die eingeschränkte Wahrnehmung von Novizinnen und Novizen wurde ebenfalls im Rahmen des professional Vision-Ansatzes (Berliner 1991) herausgearbeitet und von Stürmer et al. (2013) empirisch gestützt: Die Ergebnisse dieser Untersuchungen zeigen, dass Novizinnen und Novizen im Vergleich zu erfahrenen Lehrkräften Schwierigkeiten haben, relevante bzw. problematische Situationen zu identifizieren, diese zu interpretieren und anhand theoretischer Informationen zu erklären sowie künftige Wirkungen vorherzusagen.

Die bisher dargestellten Annahmen und Befunde weisen zusammenfassend u.a. darauf hin, dass eine Analyse von Argumentationsstrukturen allein keine vollständigen Aussagen zur Qualität der Denkprozesse zulässt (Duncan und Chinn 2016; Kiemer und Kollar 2018), da Argumentationen auch zur Bestätigung bereits vorhandener Wissensbestände genutzt werden können (Walton und Macagno 2015; Shavelson 2018). Da diese Form der Argumentation grundsätzlich von den meisten Personen beherrscht wird (Mercier und Sperber 2011), ist eine Differenzierung der genutzten Informationen notwendig, die Aussagen darüber zulässt, womit Personen ihre Behauptungen rechtfertigen (Duncan und Chinn 2016). Die Nutzung von Informationen bei der Auseinandersetzung mit Situationen wird dementsprechend als weiterer Indikator kritischer Denkprozesse betrachtet.

\subsubsection{Informationen und Informationsintegration}

Lehrkräfte können verschiedene Informationen nutzen, um sich pädagogische Situationen zu erklären. Dazu zählen u. a. eigene Erfahrungen und auch wissenschaftliches Wissen (Shulman 1987; Bromme und Tillema 1995; Cochran-Smith und Lytle 1999). Seit einigen Jahren wird die Nutzung von Informationen durch Lehrkräfte erneut unter dem Stichwort Evidenzorientierung im Bildungsbereich aufgegriffen (Prenzel 2010; Hartmann et al. 2016). Im Mittelpunkt dieser Debatte steht die aus der evidenzbasierten Medizin abgeleitete Idee, professionelle Entscheidungen nicht nur auf der Basis eigener Erfahrungen und Überzeugungen zu treffen, sondern dabei ebenso wissenschaftliche Informationen zu berücksichtigen (Sackett et al. 1996; Trempler et al. 2015; Bauer et al. 2017). Daraus kann geschlussfolgert werden, dass nicht die eine Information für die kritische Auseinandersetzung mit einer Situation und letztlich der Ableitung einer Entscheidung wichtig ist, sondern die Wahrnehmung alternativer Informationen und alternativer Erklärungen (Moshman und Tarricone 2016). Es geht also weniger darum, eine Information zu priorisieren, sondern um das Suchen, Rechtfertigen, Kombinieren oder Verwerfen verschiedener Erklärungs- 
modelle. So kann eine wiederholte unsystematische Beobachtung einer pädagogischen Situation ebenso eine Grundlage für eine Auseinandersetzung und Schlussfolgerung darstellen wie ein empirischer Befund, der in einer wissenschaftlichen Fachzeitschrift veröffentlicht wurde. Die Vorstellung, wissenschaftliches Wissen allein könne bestehende, eventuell subjektive und fehlerhafte Konzepte ersetzen, muss als Fehlvorstellung einer Informationsintegration angesehen werden (Stark 2017).

Chinn et al. (2011) unterscheiden grundsätzlich zwischen internen und externen Informationen. Interne Informationen umfassen Beobachtungen, Erfahrungen und Meinungen. Beobachtungen stellen unsystematische Betrachtungen eines Phänomens dar, die sachlich beschrieben und nicht interpretiert werden. Erfahrung und Meinung sind Sammelbegriffe für verschiedene Mechanismen, wie Introspektion, Intuition und Inferenz, die idiosynkratisch, implizit und nicht validierbar sind (Muis et al. 2006; Chinn et al. 2011). Die Nutzung interner Informationen bei der Auseinandersetzung mit Situationen wird daher oft mit subjektiven Verzerrungen konnotiert. Das kann damit begründet werden, dass erfahrungsbasierte Informationen vorwiegend in der Vergangenheit angeeignet wurden und durch selektive Wahrnehmung beeinträchtigt sein können (Chinn et al. 2011). Zudem werden erfahrungsbasierte Informationen durch das Erleben von episodischen Ereignissen angereichert, jedoch nicht systematisch verarbeitet und evaluiert (Gruber 1999). Insbesondere bei angehenden Lehrkräften kann die schon erwähnte erhöhte kognitive Belastung die Wahrnehmung und Verarbeitung in Situationen stark einschränken und verzerren (Stürmer et al. 2013; Kim und Klassen 2018).

Externe Informationen werden nicht durch die jeweilige Person konstruiert, sondern von außen an die Person herangetragen oder von ihr beschafft. Sie umfassen Formate wie Lehrbücher, Ratgeber und Expertenaussagen (Chinn et al. 2011). Die Nutzung externer Informationen für die Auseinandersetzung mit bestimmten Problemstellungen kann die Gefahr der unkritischen Anwendung in sich bergen (Sinatra et al. 2014): Dies könnte dadurch erklärt werden, dass externe Informationen oft genutzt werden, wenn Personen eher wenig Wissen auf einem Gebiet haben und dementsprechend auch keine Möglichkeit besitzen, die Validität der Quelle zu beurteilen, auf die sie sich verlassen (Hendriks et al. 2015). Letzteres konnte auch bei Universitätsstudierenden empirisch bestätigt werden, die wissenschaftliche Berichte unabhängig von ihrer Validität unkritisch zur Kenntnis nahmen und die Wissensbehauptungen in den Texten als wahr akzeptierten (Bråten et al. 2014). Weiterhin erzeugen Expertinnen und Experten bei Laien in vielen Fällen natürliche Autorität, die nicht hinterfragt wird (Latifan und Bashash 2004; Sinatra et al. 2014).

Eine Verbindung von internen und externen Informationen wird als Voraussetzung kritischer Denkprozesse beschrieben, weil dadurch bereits vorliegende Konzepte und Überzeugungen validiert, korrigiert sowie durch externe Informationen ergänzt werden können (Bromme et al. 2010). Die Nutzung verschiedener Informationen und deren sorgfältige Analyse erhöhen zudem die Sicherheit und Akkuratheit des Wissens (Kuhn 1991) und fördern das Verstehen und Schlussfolgern in spezifischen Situationen (Moshman und Tarricone 2016).

Die Befundlage zur Fähigkeit (angehender) Lehrkräfte, verschiedene Informationen in kritischen Auseinandersetzungsprozessen zu pädagogischen Situationen zu nutzen, erscheint bislang relativ dürftig (Stürmer et al. 2013). Einzelstudien weisen 
darauf hin, dass angehende Lehrkräfte Wissen nicht auf Situationen übertragen bzw. anwenden können (Stark 2005; Wagner et al. 2014) oder nur ihre eigenen internen Informationsbestände als Erklärungsgrundlage nutzen (Bromme und Tillema 1995; Kim und Klassen 2018; Kiemer und Kollar 2018). Oftmals werden diese Befunde zudem anhand von Szenarien, artifiziellen Lernumgebungen und Vignetten erzeugt (Stark et al. 2010; Trempler et al. 2015; Wenglein et al. 2015), die kaum Aussagen über die Auseinandersetzungsprozesse im realen Feld zulassen. Gerade solche real situierten und selbst ausgewählten Situationen bilden allerdings die Voraussetzung für die Entstehung persönlicher Relevanz und damit der kritischen und systematischen Auseinandersetzung mit unterschiedlichen Informationen.

\subsection{Motivationale Komponenten und authentische Situationen}

Die Auseinandersetzung mit komplexen Situationen und Informationsbeständen wird durch persönliche Einstellungen und motivationale Zustände beeinflusst (Bråten und Ferguson 2015; Hart et al. 2009; Sinatra et al. 2014). Zu diesen persönlichen Voraussetzungen gehört insbesondere die Relevanz, die eine Person einer Situation oder einem Inhalt zuschreibt (Gregoire 2003). Je stärker eine Person sich in eine Situation involviert und von ihr betroffen fühlt, desto eher wird sie sich mit dieser und mit allen damit zusammenhängenden Informationen auseinandersetzen: So beeinflusst die persönliche Relevanz die Tiefe und Form der Auseinandersetzung und führt zu tieferen Verstehens- und Elaborationsprozessen sowie tieferen Strategien der Auseinandersetzung wie dem kritischen Denken (Krapp 1999; List und Alexander 2017).

Damit Personen eine Situation als relevant erachten, sind insbesondere authentische Situationen, so genannte hot contexts von großer Bedeutung (Gregoire 2003, S. 150). Aus der Forschung zum situierten Lernen ist bekannt, dass Lernen sehr gut gefördert werden kann, wenn es in einer Situation stattfindet, die dem Anwendungskontext nahekommt (Renkl et al. 1996), da hierdurch Wissen, das bereits in Form von Repräsentationen vorliegt, auf reelle Situationen übertragen bzw. darin aktiviert wird. Die Art der Situationen bestimmt, wann welches Wissen und welche Kompetenzen angewandt werden und trägt zu deren Entwicklung/Erwerb bei (Gräsel 1997; Gräsel et al. 2006). In diversen Forschungsbereichen konnte bereits nachgewiesen werden, dass Situationen, mit denen Personen sich identifizieren können und die ihrer Lebenswelt entsprechen, die Tiefe der Auseinandersetzung beeinflussen. Dies gilt auch für Prozesse des wissenschaftlichen Argumentierens (Fischer et al. 2014), des Textverständnisses (List und Alexander 2017) und des kritischen Denkens (Abrami et al. 2015).

\subsection{Kritisches Denken und Argumentation in der Lehrerbildung}

Obwohl es möglich ist, dass verschiedene Informationen, wie Erfahrungen aus der eigenen Schulzeit und Wissen, das im Studium erworben wird, stark miteinander agieren und eventuell verschmelzen (Cochran-Smith und Lytle 1999; Chinn et al. 2011), kann es zu einer separaten Speicherung von Informationen entsprechend der Kontexte kommen, in denen sie erworben werden (bspw. Schulkontext, Universi- 
tät, Freizeit) (Clark und Linn 2013). Letzteres wird von Neuweg (2011, S. 595) als Differenzthese beschrieben. Eine systematische Verknüpfung von Informationen, die in verschiedenen Bereichen angeeignet werden (bspw. in universitären und schulischen Kontexten) sollte daher gefördert werden. Um eine Aussage darüber treffen zu können, wie angehende Lehrkräfte Informationen in ihrer Auseinandersetzung mit pädagogischen Situationen verknüpfen und ob sie dies in einer kritischen und abwägenden Weise tun, benötigen Studierende Möglichkeiten auf reale Situationen zurückzugreifen. Solche Möglichkeiten sind bspw. dann gegeben, wenn Lehramtsstudierende sich in einer Praxisphase befinden: Während der Praxisphasen werden die Studierenden in Handlungen involviert, müssen (teilweise unter Druck) Entscheidungen treffen und entwickeln Emotionen (Neuweg 2015). In diesen authentischen Kontexten kommt es nicht zu einer Vereinfachung komplexer Kontextfaktoren und Zusammenhänge, so dass Studierende selbst Problemfelder identifizieren, Fragestellungen generieren, Komplexitäten und Mechanismen verstehen und erklären und eventuell kontrastierende Erklärungsmodelle erkennen (Klopp und Stark 2016). Dabei werden im besten Fall bereits bestehende Wissensbestände aktiviert und/oder erweitert, was zu einem tieferen Verständnis der Situation führt (Hübner et al. 2007). Sie erhalten hier die Möglichkeit, sich unter universitärer Betreuung mit den Erfahrungen, die sie in authentischen Situationen machen, kritisch und anhand bereits bestehenden Wissens auseinanderzusetzen (Mertens und Gräsel 2018). Die Besonderheit der Lerngelegenheit Praxisphase liegt in einer doppelten Lernsituation, da die Studierenden sich gleichzeitig in einer universitären und einer realen schulischen Lernsituation befinden, in der unterschiedliche Handlungslogiken, Mindsets und epistemische Kriterien existieren (Kuhn 2009; Shavelson 2018). Die Fähigkeit sich kritisch mit Situationen auseinanderzusetzen kann dabei als Praktik der scientific community verstanden werden und sollte dementsprechend im universitären Bereich gelehrt werden (Klopp und Stark 2016), während praktische Erfahrungen im schulischen Umfeld erworben werden. Diese enge Verzahnung praktischer Erfahrungen und kritischer Auseinandersetzungen kann als großes Potential für die Verbindung von Theorie und Praxis angesehen werden.

Auf angehende Lehrkräfte bezogen kann das bedeuten, dass reale pädagogische Situationen, die potentiell im späteren beruflichen Kontext erneut vorkommen, eine hohe Relevanz hervorrufen und dazu beitragen, dass eventuell bereits angeeignetes theoretisches Wissen aktiviert wird. Dies ist die Voraussetzung für eine weiterführende systematische Verarbeitung.

\section{Ziele und Fragestellungen}

Kritisches Denken ist eine relevante Fähigkeit, die Lehrkräfte ihren Schülerinnen und Schülern vermitteln und die sie selbst im Sinne einer evidenzorientierten Praxis nutzen sollten, um ihre eigenen Entscheidungen und ihr professionelles Handeln zu begründen. Kritisches Denken zeigt sich in Argumentationen - bspw. wenn gerechtfertigte Annahmen aufgestellt, gegeneinander abgewogen und zur Ableitung einer Schlussfolgerung genutzt werden. Die Wahrnehmung und Darstellung verschiedener Positionen weist auf eher kritische Denkprozesse hin, während einseitige Denkpro- 
zesse - also das Vertreten einer Position bei der Auseinandersetzung - mit geringerer Wahrscheinlichkeit zu einem Aufbau neuen Wissens und alternativer Schlussfolgerungen führen.

Vor dem Hintergrund, dass unterrichtliche Handlungen stark von Annahmen und vorherrschenden Einstellungen von Lehrkräften beeinflusst werden, dass Lehrkräfte oft ungünstige Einstellungen gegenüber wissenschaftlichen Theorien zeigen (Stark et al. 2010) und dass Lehrkräfte es bevorzugen aus der Praxis gewonnene Erkenntnisse als Basis für Entscheidungen zu pädagogischen Instruktionen und Lernen zu nutzen (Bråten und Ferguson 2015), scheint die zweiseitige Argumentation besonders relevant für die pädagogische Praxis. Unsere erste Fragestellung lautet daher:

1. Argumentieren Lehramtsstudierende bei der schriftlichen und individuellen Auseinandersetzung mit pädagogischen Situationen und wenn ja, welche Argumentationsstruktur weisen die schriftlichen Auseinandersetzungen angehender Lehrkräfte mit pädagogischen Situationen auf?

Neben der Argumentationsstruktur spielt auch die Art der genutzten Informationen eine entscheidende Rolle für die Analyse von Denkprozessen, denn die Nutzung einer Informationsbasis und/oder die ausschließliche Nutzung interner oder externer Informationen kann zu Verzerrungen oder Fehlkonzepten führen. Wenn allerdings bei der Auseinandersetzung mit einem Inhalt oder einer Situation verschiedene Informationen als Alternativen wahrgenommen und genutzt werden, können alternative und gerechtfertigte Schlussfolgerungen abgeleitet werden. Letzteres ist ebenfalls ein Indikator kritischen Denkens. Unsere zweite Fragestellung lautet daher:

2. Welche Informationen nutzen Lehramtsstudierende bei der Auseinandersetzung mit pädagogischen Situationen?

Entsprechend der theoretischen Annahmen stehen zweiseitige Argumentationsstrukturen und die Integration von internen und externen Informationen in einem Zusammenhang und können als Indikatoren kritischer Denkprozesse betrachtet werden. Wir fragen uns daher drittens:

3. Gibt es einen Zusammenhang zwischen der zweiseitigen Argumentationsstruktur und der Integration interner und externer Informationen?

\section{Methodik}

\subsection{Stichprobe und Datenquellen}

Für die Beantwortung unserer Fragestellungen analysierten wir 87 schriftliche Auseinandersetzungen mit pädagogischen Situationen von Masterstudierenden (63\% weiblich; $M_{\text {Alter }}=26,41, S D_{\text {Alter }}=2,59$, unterschiedliche Fächerkombinationen). Alle Studierenden absolvierten zum Zeitpunkt der Erstellung der Einträge eine verlängerten Praxisphase (sechs Monate) an unterschiedlichen Schulen, strebten das Lehramt an Gesamtschulen und Gymnasien an und befanden sich hauptsächlich im zweiten und dritten Mastersemester $(M=2,64 ; S D=0,85)$. Wir können demnach 
davon ausgehen, dass einführende Vorlesungen zur pädagogischen Diagnostik sowie zum Lehren und Lernen bereits besucht wurden und somit Grundkenntnisse in diesen Bereichen bestanden. Die Studierenden wurden aus sechs Seminarkursen rekrutiert, denen sie zufällig zugewiesen wurden; ein Großteil (90\%) der Studierenden nahm an der Studie teil, so dass eine Positivselektion weitgehend ausgeschlossen werden kann.

Die Erstellung der schriftlichen Auseinandersetzungen stellt einen Teil der benoteten universitären Prüfungsleistung dar. Alle Studierenden stimmten einer Nutzung ihrer Einträge für Forschungszwecke zu.

\subsection{Instruktionale Vorbereitung}

Während der schulischen Praxisphase bearbeiteten die Studierenden folgende schriftliche Aufgabe:

Suchen Sie sich eine für Sie relevante pädagogische Situation (im Rahmen des Schulbetriebs oder des Unterrichts) aus. Beschreiben Sie Ihre Situation möglichst präzise und skizzieren Sie, warum diese Situation für Sie relevant ist. Erklären Sie die Situation vor dem Hintergrund von Theorien und Befunden, Erfahrungen und Beobachtungen. Ziehen Sie Schlussfolgerungen in Bezug auf Ihr professionelles Wissen (bspw. Was ist neu und wo benötigen Sie noch mehr Wissen?) und auf Ihr professionelles Handeln in (künftigen) Situationen, die der beschriebenen nahe kommen (bspw. Wie definieren Sie ihre Rolle und wie würden Sie sich verhalten wollen?). Nennen Sie die benutzten Informationen im Text und in Form einer Bibliografie.

Die schriftliche Auseinandersetzung mit pädagogischen Situationen wurde mit den Studierenden über zwei Tage (insg. 12h) anhand eines von Schellenbach-Zell et al. (2018) entwickelten Reflexionsmodells eingeübt: Darin wurden die Wahrnehmung und Beschreibung einer Situation, die Erklärung der ausgewählten Situation anhand verschiedener Informationen sowie das Ableiten von Schlussfolgerungen in Bezug auf ihr professionelles Wissen und ihr Handeln in künftigen Situationen anhand eines Videobeispiels geübt. Dadurch konnte sichergestellt werden, dass alle Elemente in der schriftlichen Auseinandersetzung während der Praxisphase vorhanden waren. Die schriftlichen Auseinandersetzungen waren im Schnitt drei Seiten lang.

\subsection{Auswertungsstrategie und Kodierschema}

Allgemeines Vorgehen Für die Kodierung der vorliegenden schriftlichen Auseinandersetzungen wählten wir das Verfahren zur Analyse verbaler Daten von Chi (1997; verbal analysis). Das Verfahren fokussiert darauf, die Repräsentation von Wissen zu verstehen und kognitive Prozesse sowie deren Änderungen über die Zeit abzubilden. Als Grundlage dafür dienen Lerntheorien, die die Lösung komplexer und schlecht strukturierter Probleme adressieren. Eine theoretische Nähe zu den in unserer Studie genutzten Konstrukten, wie dem kritischen Denken und der Argumentation, ist dementsprechend gegeben. Wie Chi (1997) ausführt, bestehen einige 
Gemeinsamkeiten zu anderen qualitativen Auswertungsverfahren, bspw. zur qualitativen Inhaltsanalyse: Beide Verfahren arbeiten mit komplexen Analyseeinheiten, das umfangreiche Material wird zergliedert und schrittweise bearbeitet, es wird theoriegeleitet am Material ein Kategoriensystem entwickelt sowie die Analyseaspekte vorher festlegt (Kuckartz 2010, S. 93). Auch sind Ähnlichkeiten der verbal analysis zur Diskursanalyse auffindbar (Allolio-Näcke 2010), deren Fokus jedoch auf Auseinandersetzungen mehrerer Personen und ganzen Textsequenzen liegt. Wir gehen davon aus, dass die von uns analysierten Texte das Produkt eines kognitiven Diskurses innerhalb einer Person darstellen. Die Analyse verbaler Daten nach Chi (1997) schien uns vor diesem Hintergrund am besten geeignet die vorliegenden schriftlichen Auseinandersetzungen zu analysieren.

Die Analyse verbaler Daten kann insgesamt acht Schritte umfassen, die bedingt durch den oftmals explorativen Charakter qualitativer Auswertungsverfahren nicht immer vollständig und nicht zwingenderweise in einer festgelegten Reihenfolge angewandt werden (Chi 1997). Hierzu gehören (1) die Reduktion und die (2) Segmentierung des Materials, (3) die Festlegung eines Kodiersystems, (4) die Operationalisierung der vorliegenden Daten entsprechend der festgelegten Kodiereinheiten, (5) die Kennzeichnung der Kodiereinheiten in den Daten, (6) die Darstellung von Strukturen zwischen den Kodiereinheiten, die (7) Interpretation der Strukturen sowie die (8) Wiederholung einzelner Schritte falls notwendig.

Reduktion und Segmentierung des Materials Die schriftlichen Auseinandersetzungen waren entsprechend der Instruktion in drei Teile untergliedert (Beschreibung, Erklärung, Schlussfolgerung). Zunächst wurde das Material um alle Teile reduziert, die zur Beantwortung der Fragestellungen nicht benötigt wurden. Anschließend wurde das Material von zwei unabhängigen Personen segmentiert und in drei Paragraphen gegliedert: Erklärung, Schlussfolgerung, Bibliografie. In Anlehnung an Chi (1997, S. 284) wurde eine recht hohe Granularität für die Segmentierung gewählt, um die Komplexität der Forschungsfragen abzubilden und ganze Argumentationsketten beizubehalten. Streitfälle (etwa wenn die einzelnen Segmente nicht klar unterscheidbar waren) wurden konsensuell diskutiert.

Festlegung der Kodiereinheiten und Operationalisierung Entsprechend der theoretischen Konstrukte wurden verschiedene Teile einer Argumentation sowie verschiedene Informationsarten als Kodiereinheiten gewählt. Dementsprechend wurden im Erklärungsteil Argumente und Gegenargumente kodiert. Anschließend wurde im Schlussfolgerungsteil kodiert, ob ein Reasoning (im Sinne einer argumentbasierten Schlussfolgerung) vorliegt. Hierfür war es notwendig, die zuvor kodierten Argumente und Gegenargumente mit den Äußerungen in der Schlussfolgerung abzugleichen. Alle Kodierschritte wurden durch zwei unabhängige Beurteilerinnen durchgeführt. Die Beurteilerübereinstimmung wurde an $50 \%$ des Materials berechnet (Beispiele und Reliabilitäten siehe Tab. 1).

Argument Ein Argument stellt eine Behauptung dar, die durch interne und/oder externe Informationen gerechtfertigt wird (Zohar und Nemet 2002). Die Kodierung eines Arguments erfolgte entsprechend einer idea bei Chi (1997). Diese umfasst 
Tab. 1 Beispiel für eine vollständige Argumentationsstruktur

\begin{tabular}{|c|c|c|}
\hline $\begin{array}{l}\text { Situation } \\
\text { Ein/e Student/ir } \\
\text { der fünften Jah } \\
\text { Schülerexperim }\end{array}$ & $\begin{array}{l}\text { rgangsstufe } \\
\text { lent und ein }\end{array}$ & $\begin{array}{l}\text { Em Biologieunterricht einer Lehrkraft auseinander, die in zwei Klassen } \\
\text { ermittlung desselben Inhalts unterschiedliche Methoden - einmal ein } \\
\text { inen Lehrervortrag - anwendet }\end{array}$ \\
\hline Kodiereinheit & Cohens $\kappa$ & Beispielaussagen \\
\hline Argument & 1,00 & $\begin{array}{l}\text { „Manfred Hesse befasste sich mit der Untersuchung von dem biolo- } \\
\text { gischen Interesse von Schülern weiterführender Schulen. Untersucht } \\
\text { wurde unter anderem die Auswirkungen der experimentellen Kompo- } \\
\text { nente des Biologieunterrichts auf das Interesse der Schüler. Untersucht } \\
\text { wurde der Verlauf der Interesse vom 5. bis zum 9. Schuljahr. Die Er- } \\
\text { gebnisse aus der Befragung mittels Fragebögen zeigten, dass Schüler } \\
\text { und Schülerinnen großen Wert auf eigene, experimentelle Tätigkei- } \\
\text { ten legten. In allen Schuljahren wurde ein überdurchschnittlicher Wert } \\
\text { von } 2,5 \text { erreicht (Item: Schülerversuch; die Skala lag zwischen 0-4; } \\
\text { 0= gar kein Interesse, } 4=\text { sehr großes Interesse). (...) Weiterhin wurde } \\
\text { anhand von Befragungen aufgezeigt, dass wegen durchgeführter Schü- } \\
\text { lerexperimente die rückwirkende Beurteilung des Unterrichts durch die } \\
\text { Schüler erheblich positiver ausfiel. Die Bewertung von Lehrerversu- } \\
\text { chen/Demonstrationsexperimenten fiel im Durchschnitt schlechter ab als } \\
\text { Schülerversuche.“ (Z: } 102-115, \text { GW30JN) }\end{array}$ \\
\hline $\begin{array}{l}\text { Gegen- } \\
\text { argument }\end{array}$ & 0,63 & $\begin{array}{l}\text { „Als Grund für diese nur theoretische Einführung des Themas nannte die } \\
\text { Lehrerin, dass sie den Schülern nicht zu traue eigenständig und vorsich- } \\
\text { tig mit einem scharfen Messer/Skalpell zu arbeiten. (...) Wie das obige } \\
\text { Beispiel zeigt, sind manche Experimente, auch wenn sie scheinbar sehr } \\
\text { einfach konzipiert sind, mit manchen Klassen schwierig umzusetzen, da } \\
\text { fehlende Kompetenzen (Umgang mit Messern/Skalpellen) Gefahrquellen } \\
\text { sind.“ (Z: 85-125; GW30JN) }\end{array}$ \\
\hline $\begin{array}{l}\text { Reasoning } \\
\text { mit Bezug } \\
\text { zu Argu- } \\
\text { ment und } \\
\text { Gegenar- } \\
\text { gument }\end{array}$ & 0,77 & $\begin{array}{l}\text { „Nach den bisherigen Studien und Erkenntnissen bezüglich der Ein- } \\
\text { bindung von Experimenten in den Biologieunterricht werde ich als } \\
\text { Biologielehrerin versuchen solche eigentlich relativ einfach Experi- } \\
\text { mente bzw. praktischen Sequenzen im Biologieunterricht so oft es geht } \\
\text { in den Unterricht mit einzubinden. Wenn die Umsetzung mit einigen } \\
\text { Schülern bzw. Klassen schwierig ist hinsichtlich des Sicherheit, würde } \\
\text { ich auf ein Schülerexperiment verzichten jedoch einen Lehrerversuch/ } \\
\text { Demonstrationsexperiment einplanen. Diese sind, wie Hesse zeigte, zwar } \\
\text { nicht so beliebt wie Schülerversuche, wirken sich jedoch motivieren- } \\
\text { der auf die Schüler aus als Unterricht ohne Experimente.“ (Z: 129-136; } \\
\text { GW30JN) }\end{array}$ \\
\hline
\end{tabular}

eine ganze Sinneinheit, die auf dem semantischen Level erfasst wird, damit der gesamte Inhalt transportiert wird und Redundanzen vermieden werden. Semantische Sinneinheiten umfassen zumeist mehrere Sätze mit ganzen Argumentations-/ Erklärungsketten (Chi 1997; Kuhn et al. 2016).

Gegenargument Ein Gegenargument widerspricht einer zuvor dargestellten Position und wird ebenfalls durch Informationen gerechtfertigt. Argumente und Gegenargumente werden implizit oder explizit mit einem ,weil“ eingeleitet. Alle Argumente und Gegenargumente des Textes wurden einzeln identifiziert und ggf. unter den beiden Beurteilerinnen bei Nichtübereinstimmung bis zur Konsensfindung diskutiert. In einem zweiten Schritt wurde für den gesamten Text ein dichotomer Code $(0=$ nicht vorhanden, 1 = vorhanden) vergeben. 
Tab. 2 Kategorien interner und externer Informationen und Kodierregeln

\begin{tabular}{|c|c|c|}
\hline & Kodierregeln & Beispielaussage \\
\hline $\begin{array}{l}\text { Interne } \\
\text { Informa- } \\
\text { tionen }\end{array}$ & \multicolumn{2}{|c|}{$\begin{array}{l}\text { In dieser Kategorie werden alle Informationen kodiert, die aus der Person selbst kommen. } \\
\text { Hierzu gehören unsystematische Beobachtung, Erfahrung und Meinung. }\end{array}$} \\
\hline Beobachtung & $\begin{array}{l}\text { Eine sachlich dargestell- } \\
\text { te Beschreibung einer } \\
\text { unsystematisch beobach- } \\
\text { teten Situation. Aussa- } \\
\text { gen, die kodiert wurden: } \\
\text { „ich habe gesehen“, } \\
\text { „ich habe beobachtet“, } \\
\text { „mir fiel auf“, ,ich habe } \\
\text { wahrgenommen“ usw }\end{array}$ & $\begin{array}{l}\text { „Bezüglich der Aufgabenstellung und Materialien konnte im } \\
\text { Biologieunterricht einer } 8 \text {. Klasse beobachtet werden, dass } \\
\text { für die Lösung der gestellten Aufgabe das Schulbuch nicht } \\
\text { die benötigten Informationen enthielt. Erst auf mehrmalige } \\
\text { Anfragen der SuS wurde die Lehrkraft darauf Aufmerksam } \\
\text { und besorgte im Anschluss mit zwei SuS, } 10 \text { min vor Ende } \\
\text { des Unterrichts die } \\
\text { geeigneten Bücher aus dem Vorbereitungsraum.“ (SW24SS; } \\
\text { Z: 142-144) }\end{array}$ \\
\hline $\begin{array}{l}\text { Erfahrung/ } \\
\text { Meinung }\end{array}$ & $\begin{array}{l}\text { Stellen wahrgenom- } \\
\text { mene Aspekte aus der } \\
\text { Vergangenheit dar, die } \\
\text { oft mit Interpretationen } \\
\text { und Schlussfolgerungen } \\
\text { einhergehen. Aussa- } \\
\text { gen, die einen eigenen } \\
\text { Standpunkt erklären }\end{array}$ & $\begin{array}{l}\text { „Als Gegenbeispiel möchte ich (...) die Erfahrung auffüh- } \\
\text { ren, dass ich es als Schüler selbst immer enorm genoss, } \\
\text { wenn Lehrkräfte mit uns Schüler_innen zusammen lach- } \\
\text { ten. Dadurch herrschte eine sehr positive Arbeitsatmosphäre.“ } \\
\text { (GH17MS; Z: 114-116) } \\
\text { „Meiner Meinung nach hat der Schüler jedoch dieses Ange- } \\
\text { bot des Lehrers ziemlich schnell in Form seiner negativen, } \\
\text { ablehnenden Haltung abgeschlagen und keinen Willen ge- } \\
\text { zeigt, seine Mitarbeit zu verbessern. Dadurch finde ich die } \\
\text { Vergabe der schlechteren Note durch den Lehrer durchaus } \\
\text { angemessen.“(KJ07JA; 161-164) }\end{array}$ \\
\hline
\end{tabular}

Externe In dieser Kategorie werden alle Informationen kodiert, die von außen an die Person heranInforma- getragen oder von ihr beschafft wurden

tionen

Handbuch/ Expliziter Verweis auf

Lehrbuch/ externe Informationen.

Sammelband Diese können im

Emp. Fließtext oder in einer

Zeit- Klammer genannt

schrif- werden. Wichtig ist, dass

tenbeitrag deutlich wird, zu

sonst. welcher Information die

externe Aussage gehört

Informa-

tion

Allgemeiner Hierzu zählen Aussa-

Verweis gen, bei denen deutlich

Bezug zu externen In-

formationen genommen, die aber nicht explizit genannt werden

Experten- Übernahme von Wissen aussage und Einstellungen durch die Behauptung(en) anderer Personen (Wissenschaftler, Lehrkräfte usw.). Es werden alle Aussagen kodiert, die sich explizit auf Aussagen anderer Personen beziehen
„In Anlehnung an Hattie et al. (2013) formuliert R. Wozinski (2015) drei Komponenten. Diese drei Komponenten ,feed up', ,feed back' und ,feed forward" sollen in einem guten Feedback enthalten sein (...).“(DM13SC; Z: 85-87)

„Nach meiner Literaturrecherche fand ich heraus, dass Studien gezeigt haben, dass sich ein personenzentriertes Lehrerverhalten positiv auf die SuS auswirkt." (Quelle wird nicht genannt; SA10SL; Z: 126-130)

„In der Reflektion der Stunde sagte mir der mich im Unterricht begleitende Lehrer, dass ich die für die Unterrichtsstunde geplante Thematisierung der Streitentwicklung und der Vorbeugung seiner Eskalation trotz der nicht zu Ende vorgestellten Ergebnisse der SuS hätte durchführen sollen.“ (ER07MJ; Z: 72-77) 
Reasoning Ein Reasoning stellt eine Schlussfolgerung dar, in der ein Bezug zu mindestens einem (Gegen)Argument im Erklärungsteil besteht. Dies wird über Schlagworte, Synonyme und/oder semantische Übereinstimmung zwischen Argument(en) und Schlussfolgerung ermittelt. Die Art der Schlussfolgerung wurde dichotom kodiert ( $1=$ reasoning; $0=$ kein reasoning; Moshman und Tarricone 2016). Reasoning wurde ausschließlich im Schlussfolgerungsteil kodiert.

Informationen Parallel wurden die Informationen in Anlehnung an vorgeschlagene Typologien (Chinn et al. 2011) kodiert und ausgezählt. In einem ersten Schritt wurde die Unterscheidung in interne und externe Informationen (Cohen's $\kappa=0,95$ ) vorgenommen und anschließend wurden diese Informationen noch einmal nach Art differenziert (Cohen's $\kappa=0,77$ ) (siehe Tab. 2). Die Kodierung erfolgte durch zwei unabhängige Beurteilerinnen und wurde an $47 \%$ der vorliegenden Daten validiert.

Darstellung von Strukturen zwischen den Kodiereinheiten Im Anschluss an die Kodierung wurden alle relevanten Teile einer vollständigen Argumentationsstruktur ${ }^{1}$ zu der Variable Argumentationsstruktur in drei Abstufungen aggregiert: (1) Texte, die kein Reasoning enthielten, wurden als non-argumentations eingeordnet; (2) Auseinandersetzungen, die ausschließlich Argumente und ein daraus abgeleitetes Reasoning enthielten, wurden als einseitige Argumentationen eingeordnet; und (3) Auseinandersetzungen, die Argumente, Gegenargumente sowie ein Reasoning enthielten, wurden als zweiseitige Argumentationen eingeordnet.

\section{Ergebnisse}

Die erste Fragestellung befasst sich damit, ob angehende Lehrkräfte in der schriftlichen Auseinandersetzung mit pädagogischen Situationen argumentieren und welche Struktur diese Argumentationen aufweisen. Es zeigt sich, dass insgesamt 89,9\% $(n=80)$ der schriftlichen Auseinandersetzungen eine vollständige Argumentationsstruktur aufweisen, während 10,1\% ( $n=9)$ der Auseinandersetzungen keine Argumentation beinhalten. Insgesamt 56,2\% $(n=50)$ der Auseinandersetzungen weisen eine einseitige Argumentationsstruktur auf und 33,7\% $(n=30)$ aller kodierten Einträge weisen eine zweiseitige Argumentationsstruktur auf, in der Argumente und Gegenargumente sowie ein Reasoning vorhanden sind.

Mit der zweiten Fragestellung untersuchen wir, welche Informationen Lehramtsstudierende nutzen, wenn sie sich schriftlich mit einer schulischen Situation auseinandersetzen. Insgesamt werden vor allem externe Informationen von den Studierenden verwendet - im Mittel werden je Auseinandersetzung 1,8 interne und 7 externe Informationen verwendet. Insbesondere Hand-, Lehrbüchern und Sammelbänden

\footnotetext{
1 Die Kategorie Behauptung wurde nicht als Kategorie aufgenommen, obwohl sie theoretisch zu einer vollständigen Argumentation gezählt wird, da diese sowohl implizit als auch explizit genannt werden kann und in den meisten Fällen einen Teil der Argumente darstellen (Zohar und Nemet 2002; Osborne 2010). Das Vorhandensein einer These ist daher kein Indikator einer vollständigen Argumentation oder der Argumentationsqualität.
} 
Tab. 3 Übersicht der verwendeten Informationen $($ gesamt = 770)

\begin{tabular}{lll}
\hline & \multicolumn{2}{l}{ Häufigkeiten } \\
\hline Informationsarten & Absolut & Relativ \\
Anzahl interne Informationen (insgesamt) & 157 & 20,4 \\
Beobachtung & 31 & 4,0 \\
Erfahrung/Meinung & 126 & 16,4 \\
Anzahl externe Informationen (insgesamt): & 613 & 79,6 \\
Handbuch/Lehrbuch/Sammelband & 240 & 31,2 \\
Emp. Zeitschriftenartikel & 88 & 11,4 \\
Sonstige externe Informationen (Ratgeber, Ministerialtext) & 202 & 26,2 \\
Allgemeiner Verweis & 68 & 8,8 \\
Expertenaussagen & 10 & 1,3 \\
\hline
\end{tabular}

sowie sonstigen externen Quellen (bspw. Ratgeber, Gesetzestexte, praxisorientierte Publikationen) werden für die Auseinandersetzung mit pädagogischen Situationen herangezogen. Etwa ein Fünftel aller Informationen stellen interne Informationen dar (siehe Tab. 3).

Die differenzierte Betrachtung der verwendeten Informationen innerhalb der verschiedenen Argumentationsstrukturen (siehe Tab. 4) zeigt folgendes Bild: (1) In Auseinandersetzungen ohne vollständige Argumentationsstruktur wurden vor allem Erfahrungen und Meinungen sowie sonstige externe Informationen verwendet. (2) Bei einseitigen Argumentationen zeigt sich eine häufige Verwendung von Lehr-/

Tab. 4 Übersicht verwendeter Informationen innerhalb der Argumentationsstrukturen

\begin{tabular}{|c|c|c|c|c|c|}
\hline & \multirow{2}{*}{$\begin{array}{l}\text { Non-argumen- } \\
\text { tation } \\
\text { Extern und } \\
\text { intern } \\
(n=7)\end{array}$} & \multicolumn{2}{|c|}{ Einseitige Argumentation } & \multicolumn{2}{|c|}{ Zweiseitige Argumentation } \\
\hline & & $\begin{array}{l}\text { Extern } \\
(n=21)\end{array}$ & $\begin{array}{l}\text { Intern \& ex- } \\
\text { tern } \\
(n=28)\end{array}$ & $\begin{array}{l}\text { Extern } \\
(n=7)\end{array}$ & $\begin{array}{l}\text { Intern \& } \\
\text { extern } \\
(n=22)\end{array}$ \\
\hline \multicolumn{6}{|c|}{ Interne Informationen } \\
\hline Beobachtung & 0,86 & - & 0,32 & - & 0,73 \\
\hline $\begin{array}{l}\text { Erfahrung/ } \\
\text { Meinung }\end{array}$ & 2,57 & - & 1,89 & - & 2,50 \\
\hline \multicolumn{6}{|c|}{ Externe Informationen } \\
\hline $\begin{array}{l}\text { Hand-/Lehrbuch/ } \\
\text { Sammelband }\end{array}$ & 0,57 & 2,67 & 3,36 & 2,57 & 2,86 \\
\hline $\begin{array}{l}\text { Emp. Zeitschrif- } \\
\text { tenartikel }\end{array}$ & 0,29 & 0,43 & 0,64 & 3,86 & 1,45 \\
\hline $\begin{array}{l}\text { Sonstige externe } \\
\text { Informationen }\end{array}$ & 2,86 & 1,81 & 2,39 & 2,86 & 2,55 \\
\hline $\begin{array}{l}\text { Allgemeine Ver- } \\
\text { weise }\end{array}$ & 0,43 & 0,76 & 0,82 & 1,71 & 0,55 \\
\hline Expertenaussagen & 0,29 & 0,14 & 0,07 & 0,29 & 0,05 \\
\hline
\end{tabular}

Anmerkung. Die dargestellten Werte sind gemittelte Häufigkeiten der jeweiligen verwendeten Informationen in den schriftlichen Auseinandersetzungen. Keine schriftliche Auseinandersetzung, die eine unvollständige Argumentationsstruktur (non-argumentation) aufweist, nutzt ausschließlich externe Informationen 
Handbüchern und Sammelbänden sowie sonst. externen Informationen. Wenn auch interne Informationen herangezogen wurden, dann vor allem Erfahrungen und Meinungen; Beobachtungen werden eher selten genutzt. (3) In zweiseitigen Argumentationen wurden deutlich mehr wissenschaftliche Zeitschriftenbeiträge verwendet als in einseitigen Argumentationen und non-argumentations. Dies ist vor allem dann der Fall, wenn keine Integration interner und externer Informationen stattfindet (siehe Tab. 4).

Zur Beantwortung der dritten Fragestellung wurden die Zusammenhänge zwischen Argumentationsstruktur (non-argumentation, einseitig, zweiseitig) und Art der Informationsintegration (intern, extern, extern und intern) anhand einer Kreuztabelle ermittelt. Es zeigt sich, dass zweiseitige Argumentationen im Vergleich zu einseitigen und non-argumentations stärker mit einer Verbindung von internen und externen Informationen einhergeht $\left(76 \% ; \chi^{2}(4)=16.145, p=0,003\right.$; Cramers- $\mathrm{V}=0,306$, $p=0,003)$.

\section{Diskussion}

Die Ergebnisse zur ersten Fragestellung zeigen, dass ein Großteil der Studierenden in den schriftlichen Auseinandersetzungen vollständige Argumentationen verfasst, d.h. auf der Basis verschiedener Argumente zu mindestens einer Position zu einer eigenen Schlussfolgerung gelangt ist. Ein Viertel der untersuchten Studierenden verbindet Argumente und Gegenargumente zu einer zweiseitigen Argumentation. In Bezug auf die vorliegenden Ergebnisse lässt sich schlussfolgern, dass ein Viertel der angehenden Lehrkräfte in ihren schriftlichen Argumentationen Ansätze für kritisches Denken zeigt.

Die Befunde zur zweiten Fragestellung weisen darauf hin, dass angehende Lehrkräfte in ihre schriftlichen Auseinandersetzungen vorwiegend externe Informationen einfließen lassen. Diesen Effekt konnte Kuhn (2015) bereits an Lernenden nachweisen, die in argumentativen Essays primär externe Informationen nutzten, während sie in dialogischen Settings eher auf interne Informationsbestände zurückgriffen: Sie erklärt dieses Ergebnis damit, dass Personen interne Informationen zurückhalten, wenn sie keine Relevanz ihrer eigenen Meinung und Erfahrung bei der Verfassung von argumentativen Texten sehen - möglicherweise, weil dies in universitären Settings nicht üblich ist. Unklar bleibt allerdings, ob Studierende eher solche externen Informationen heranziehen, die ihren eigenen Meinungen/Erfahrungen entsprechen ohne diese internen Quellen explizit zu erwähnen.

Die differenzierte Betrachtung der Argumentationsstruktur zeigt:

1. Non-argumentations gehen mit der Verwendung nicht-wissenschaftlicher Literatur und eher subjektiven Informationen wie Erfahrung und Meinung einher. Dies kann auf eine verringerte Argumentationsfähigkeit und/oder Schwierigkeiten bei der Recherche und Bewertung von Informationen hinweisen.

2. Einseitige Argumentationen stehen häufig in Verbindung mit Lehrbüchern, Handbüchern, Sammelbänden sowie sonstigen externen Informationen (z. B. Ratgeberliteratur). Das bedeutet, dass Studierende prinzipiell argumentieren können, je- 
doch keine Gegenposition einfließen lassen und eher Literatur verwenden, in der theoretische Ansätze auf einem abstrakten Niveau beschrieben werden. Diese Art der Auseinandersetzung impliziert möglicherweise, dass bereits bestehende interne Informationen durch entsprechend deckungsgleiche externe Informationen bestätigt werden (Hart et al. 2009).

3. Ein bemerkenswertes Ergebnis betrifft die zweiseitigen Argumentationen, die wir in den schriftlichen Auseinandersetzungen finden konnten. Diese weisen auf eine kritische Form der Auseinandersetzung hin. Wird eine Position mit wissenschaftlichen Informationen gerechtfertigt, weist das auf die Fähigkeit hin, evidenzorientiert zu argumentieren (Iordanou et al. 2016). Diese Annahme kann tendenziell durch unsere Ergebnisse gestützt werden, wonach zweiseitige Argumentationen sich durch eine auffallend höhere Nutzung von wissenschaftlichen Zeitschriftenartikeln auszeichnen. Überraschend erscheint in dem Zusammenhang, dass wissenschaftliche Zeitschriftenartikel vor allem dann genutzt werden, wenn keine internen Informationen in die Argumentation einfließen. Fließen Beobachtungen, Meinungen und Erfahrung in die Auseinandersetzungen ein, werden deutlich weniger Zeitschriftenartikel herangezogen. Hierfür bieten sich mindestens zwei Erklärungsansätze an: (1) dass interne Informationen eine ähnliche Funktion wie wissenschaftliche Zeitschriftenartikel erfüllen oder (2) dass empirische Befunde dann genutzt werden, wenn keine eigenen Erfahrungen vorliegen. Ungeklärt bleibt an dieser Stelle die Bedeutung einer zweiseitigen Argumentation, in der ausschließlich externe Informationen genutzt werden: Möglicherweise zeigen sich in dieser recht selten vertretenen Kombination Ansätze wissenschaftlicher Argumentation (Fischer et al. 2014) und das Abwägen konfligierender, wissenschaftlicher Positionen (Bauer et al. 2017). Aus universitärer Sicht sind solche Fähigkeiten erwünscht; inwieweit sie sich auch auf die Arbeit als angehende Lehrkraft im Praxisfeld auswirken, ist noch fraglich.

Entsprechend unserer Annahmen zur dritten Fragestellung korrespondieren Argumentationsstrukturen und die Informationsintegration dergestalt, dass eine zweiseitige Argumentationsstruktur mit einer häufigeren Informationsintegration einhergeht. Dieses Ergebnis kann als Hinweis darauf gewertet werden, dass kritische (zweiseitige) Auseinandersetzungsprozesse und die Integration verschiedener Informationsbestände (intern und extern) zusammenhängen.

Die dargestellten Ergebnisse sind unter Berücksichtigung einiger Limitationen vorsichtig zu interpretieren: Es handelt sich um eine kleine Stichprobe, die nicht hinreichend entsprechend ihrer Eingangsvoraussetzungen beschrieben werden kann. Die Stärke der Studie, die wir in der freien Wahl pädagogischer Situationen verorten, ist zugleich als methodische Schwäche anzumerken: Es ist noch ungeklärt, ob jede schulische Situation sich gleichermaßen für eine kritische Auseinandersetzung eignet und genügend Informationen aus unterschiedlichen Quellenarten zur Erklärung prinzipiell jeder ausgewählten Situation bereitstehen. Mit einer Vorgabe von Situationen würde allerdings die wahrgenommene persönliche Relevanz eingeschränkt werden, die als Voraussetzung für eine kritische Auseinandersetzung gesehen wird. Eine grundsätzliche Auflösung dieses Dilemmas erscheint nicht möglich - seine Implikationen sollten daher im Fortgang weiterer Forschung intensiv 
untersucht werden. Diese Limitationen insbesondere in Bezug auf die Stichprobe sowie das qualitativ-explorative Untersuchungsdesign führen zwangsläufig zu einer eher geringen Verallgemeinerbarkeit der Ergebnisse.

Mit unseren Forschungsbemühungen werfen wir einen Blick auf spontane Auseinandersetzungsprozesse angehender Lehrkräfte mit realen und selbstgewählten pädagogischen Situationen. Die Ergebnisse weisen darauf hin, dass Studierende in der Lage sind, evidenzorientiert zu argumentieren - also verschiedene wissenschaftliche Informationen für die Auseinandersetzung mit pädagogischen Situationen zu nutzen. Das kritische Abwägen verschiedener Positionen gelingt jedoch nur knapp einem Viertel der Studierenden.

Daraus ergeben sich Fragen für weiterführende quantitativ angelegte Studien, die die bisherigen Ergebnisse validieren und Zufallsbefunde ausschließen. Hierzu gehören kleinere Interventionen zur Informationsintegration und zu Fähigkeiten des kritischen Denkens und der Argumentation. Bereits existierende Ansätze zur Förderung kritischen Denkens (Abrami et al. 2015) sowie zum Multiple Source Use (List und Alexander 2017) könnten dabei als Ausgangspunkte dienen.

Zudem sollte untersucht werden, ob die von Hart et al. (2009) bestätigte selektive Nutzung von Informationen in den schriftlichen Auseinandersetzungen zu Tage tritt: Möglicherweise nutzen die Studierenden leicht verständliche Literatur (bspw. Lehrbücher und Ratgeber), um ihre Meinungen und Erfahrungen darzustellen. Obwohl diese Form oberflächlich als Nutzung ausschließlich externer Informationen erkannt wird, stellt sie durchaus eine Form der Informationsintegration dar, die bspw. anhand von stimulated recalls (Calderhead 1981) näher untersucht werden könnte.

Letztlich betrachten wir bislang ausschließlich Fähigkeiten der kritischen und informationsbasierten Argumentation - weitere Faktoren, wie Überzeugungen in Bezug auf verschiedene Formen von Wissen, die durchaus Einfluss auf die Auseinandersetzung haben, werden bisher nicht beachtet. Bråten und Ferguson (2015) konnten bereits zeigen, dass die Überzeugung dazu, woher relevantes Wissen kommt, die Auseinandersetzung mit diesem Wissen beeinflusst. Wenn also Studierende davon überzeugt sind, dass nur die Praxis relevante Fragen zu Instruktion und Lernen beantworten kann, ist die Wahrscheinlichkeit höher, dass vor allem aus der Praxis stammende Informationen verwendet werden. Überzeugungen zur Erzeugung relevanten pädagogischen Wissens in der Forschung gehen demgegenüber mit einer stärkeren Nutzung dieser Informationsbestände einher.

Es bleibt offen, ob Ansätze der kritischen, informationsbasierten Reflexion tatsächlich förderlich für praktische Entscheidungen von Lehrkräften im schulischen Kontext sind. Bewährte Reflexionsmodelle nach Schön (1983), Schön und Argyris (1996) und Korthagen (2010) ebenso wie Ansätze evidenzorientierter schulischer Praxis (Bauer et al. 2017) gehen davon aus, dass die reflektierte Nutzung von Informationen pädagogische Entscheidungen und Handlungen verbessert. Die Forschung zur Evidenzorientierung im Lehrerberuf bleibt bislang Antworten schuldig, inwieweit dies tatsächlich der Fall ist (Stark 2017). Ebenso offen bleibt, wie die Fähigkeiten der kritischen Reflexion in die Ausbildung von Lehrkräften integriert werden kann. Diese sowohl wissenschaftlich als auch praktisch relevanten Fragen könnten in weiterer anwendungsorientierter pädagogisch-psychologischer Forschung im Kontext verlängerter Praxisphasen beantwortet werden. 
Funding Open Access funding enabled and organized by Projekt DEAL.

Open Access Dieser Artikel wird unter der Creative Commons Namensnennung 4.0 International Lizenz veröffentlicht, welche die Nutzung, Vervielfältigung, Bearbeitung, Verbreitung und Wiedergabe in jeglichem Medium und Format erlaubt, sofern Sie den/die ursprünglichen Autor(en) und die Quelle ordnungsgemäß nennen, einen Link zur Creative Commons Lizenz beifügen und angeben, ob Änderungen vorgenommen wurden.

Die in diesem Artikel enthaltenen Bilder und sonstiges Drittmaterial unterliegen ebenfalls der genannten Creative Commons Lizenz, sofern sich aus der Abbildungslegende nichts anderes ergibt. Sofern das betreffende Material nicht unter der genannten Creative Commons Lizenz steht und die betreffende Handlung nicht nach gesetzlichen Vorschriften erlaubt ist, ist für die oben aufgeführten Weiterverwendungen des Materials die Einwilligung des jeweiligen Rechteinhabers einzuholen.

Weitere Details zur Lizenz entnehmen Sie bitte der Lizenzinformation auf http://creativecommons.org/ licenses/by/4.0/deed.de.

\section{Literatur}

\section{Verwendete Literatur}

Abrami, P.C., Bernard, R. M., Borokhovski, E., Waddington, D. I., Wade, A.C., \& Persson, T. (2015). Strategies for teaching students to think critically: a meta-analysis. Review of Educational Research, $85(2), 275-314$.

Alexander, P. A. (2017). Reflection and reflexivity in practice versus in theory: Challenges of conceptualization, complexity, and competence. Educational Psychologist, 52(4), 307-314.

Allolio-Näcke, L. (2010). Diskursanalyse. In G. Mey \& K. Mruck (Hrsg.), Handbuch qualitative Forschung in der Psychologie (S. 662-675). Wiesbaden: Springer.

Asterhan, C.S.C., \& Schwarz, B. B. (2016). Argumentation for learning: Well-trodden paths and unexplored territories. Educational Psychologist, 51(2), 164-187.

Bauer, J., Berthold, K., Hefter, M.H., Prenzel, M., \& Renkl, A. (2017). Wie können Lehrkräfte und ihre Schülerinnen und Schüler lernen, fragile Evidenz $\mathrm{zu}$ verstehen und $\mathrm{zu}$ nutzen? Psychologische Rundschau, 68(3), 188-192.

Berliner, D.C. (1991). Educational psychology and pedagogical expertise: New findings and new opportunities for thinking about training. Educational psychologist, 26(2), 145-155.

Berliner, D.C. (2002). Educational research: the hardest science of all. Educational Researcher, 31(8), 18-20.

Bråten, I., \& Ferguson, L.E. (2015). Beliefs about sources of knowledge predict motivation for learning inteacher education. Teaching and Teacher Education, 50, 13-23.

Bråten, I., Ferguson, L.E., Strømsø, H. I., \& Anmarkrud, Ø. (2014). Students working with multiple conflicting documents on a scientific issue: relations between epistemic cognition while reading and sourcing and argumentation in essays. British Journal of Educational Psychology, 84(1), 58-85.

Bromme, R., \& Kienhues, D. (2014). Wissenschaftsverständnis und Wissenschaftskommunikation. In T. Seidel \& A. Krapp (Hrsg.), Pädagogische Psychologie (Bd. 6, S. 55-81). Weinheim: Beltz.

Bromme, R., \& Tillema, H. (1995). Fusing experience and theory: The structure of professional knowledge. Learning and Instruction, 5(4), 261-267.

Bromme, R., Kienhues, D., \& Porsch, T. (2010). Who knows what and who can we believe? Epistemological beliefs are beliefs about knowledge (mostly) to be attained from others. In L. D. Bendixen \& F. C. Feucht (Hrsg.), Personal epistemology in the classroom (S. 163-194). Cambridge: Cambridge University Press.

Buehl, M.M., Alexander, P.A., Murphy, P.K., \& Sperl, C.T. (2001). Profiling persuasion: The role of beliefs, knowledge, and interest in the processing of persuasive texts that vary by argument structure. Journal of Literacy Research, 33(2), 269-301.

Calderhead, J. (1981). Stimulated recall: A method for research on teaching. British Journal of Educational Psychology, 51(2), 211-217. 
Chaiken, S. (1980). Heuristic versus systematic information processing and the use of source versus message cues in persuasion. Journal of Personality and Social Psychology, 39(5), 752-766.

Chi, M.T.H. (1997). Quantifying qualitative analyses of verbal data: A practical guide. The Journal of the Learning Sciences, 6(3), 271-315.

Chinn, C. A., Buckland, L.A., \& Samarapungavan, A. (2011). Expanding the dimensions of epistemic cognition: Arguments from philosophy and psychology. Educational Psychologist, 46(3), 141-167.

Clark, D. B., \& Linn, M. (2013). The knowledge integration perspective. In S. Vosniadou (Hrsg.), International handbook of research on conceptual change (S. 520-537). New York: Routledge.

Cochran-Smith, M., \& Lytle, S.L. (1999). Relationships of knowledge and practice: Teacher learning in communities. Review of Research in Education, 24(1), 249-305.

Common Core Standards Initiative (2010) Common Core Standards for English Language Arts \& Literacy in History/Social Studies, Science, and Technical Subjects (2010). http://www.corestandards.org/wpcontent/uploads/ELA_Standards1.pdf. Zugegriffen: 12. Juni 2017.

Crowell, A., \& Kuhn, D. (2014). Developing dialogic argumentation skills: A 3-year intervention study. Journal of Cognition and Development, 15(2), 363-381.

Duncan, R. G., \& Chinn, C. A. (2016). New directions for research on argumentation: insights from the AIR framework for epistemic cognition. Zeitschrift für Pädagogische Psychologie, 30(2-3), 155-161.

Farrell, T.S.C. (2012). Reflecting on reflective practice: (Re)visiting Dewey and Schön. TESOL Journal, $3(1), 7-16$.

Fischer, F., Kollar, I., Stegman, K., \& Wecker, C. (2013). Toward a script theory of guidance in computersupported collaborative learning. Educational Psychologist, 48, 56-66.

Fischer, F., Kollar, I., Ufer, S., Sodian, B., Hussmann, H., Pekrun, R., Neuhaus, B., Dorner, B., Pankofer, S., Fischer, M. R., Strijbos, J.-W., Heene, M., \& Eberle, J. (2014). Scientific reasoning and argumentation: Advancing an interdisciplinary research agenda in education. Frontline Learning Research, 5 , $28-45$.

Fisher, A. (2011). Critical thinking: an introduction. Cambridge: University Press.

Gräsel, C. (1997). Problemorientiertes Lernen: Strategieanwendung und Gestaltungsmöglichkeiten. Göttingen: Hogrefe.

Gräsel, C., Fussangel, K., \& Parchmann, I. (2006). Lerngemeinschaften in der Lehrerfortbildung. Zeitschrift für Erziehungswissenschaft, 9(4), 545-561.

Gregoire, M. (2003). Is it a challenge or a threat? A dual-process model of teachers' cognition and appraisal processes during conceptual change. Educational Psychology Review, 15(2), 147-179.

Gruber, H. (1999). Erfahrung als Grundlage kompetenten Handelns. Bern: Huber.

Hart, W., Albarracín, D., Eagly, A. H., Brechan, I., Lindberg, M. J., \& Merrill, L. (2009). Feeling validated versus being correct: A meta-analysis of selective exposure to information. Psychological Bulletin, 135(4), 555-588.

Hartmann, U., Decristan, J., \& Klieme, E. (2016). Unterricht als Feld evidenzbasierter Bildungspraxis? Zeitschrift für Erziehungswissenschaft, 19(1), 179-199.

Hendriks, F., Kienhues, D., \& Bromme, R. (2015). Measuring laypeople's trust in experts in a digital age: The muenster epistemic trustworthiness inventory (METI). PLoS ONE, 10(10), 1-20.

Hofer, B.K. (2016). Epistemic cognition as a psychological construct: Advancements and challenges. In J. Greene, W. A. Sandoval \& I. Bråten (Hrsg.), Handbook of epistemic cognition (S. 31-50). New York: Routledge.

Hübner, S., Nückles, M., \& Renkl, A. (2007). Lerntagebücher als Medium des selbstgesteuerten Lernens - Wie viel instruktionale Unterstützung ist sinnvoll. Empirische Pädagogik, 21(2), 119-137.

Iordanou, K. (2010). Developing argument skills across scientific and social domains. Journal of Cognition and Development, 11(3), 293-327.

Iordanou, K., Kendeou, P., \& Beker, K. (2016). Argumentative reasoning. In J. Greene, W. A. Sandoval (Hrsg.) Handbook of epistemic cognition (S. 31-50). New York: Routledge.

Kiemer, K., \& Kollar, I. (2018). Evidence-based reasoning of pre-service teachers-a script perspective. Vortrag auf der 13. International Conference of the Learning Sciences, London, 23.-30. Juni.

Kim, L.E., \& Klassen, R. M. (2018). Teachers' cognitive processing of complex school-based scenarios: Differences across experience levels. Teaching and Teacher Education, 73, 215-226.

Klopp, E., \& Stark, R. (2016). Persönliche Epistemologien - Elemente wissenschaftlicher Kompetenz. In A.-K. Mayer \& T. Rosman (Hrsg.), Denken über Wissen und Wissenschaft. Epistemologische Überzeugungen als Gegenstand psychologischer Forschung (S. 39-69). Lengerich: Pabst Science Publishers.

Korthagen, F. A. J. (2010). Situated learning theory and the pedagogy of teacher education: Towards an integrative view of teacher behavior and teacher learning. Teaching and Teacher Education, 26, 98-106. 
Korthagen, F. A. J., \& Wubbels, T. (2008). Learning from Practice. In F. A. J. Korthagen, J. Kessels, B. Koster, \& B. Lagerwerf (Hrsg.), Linking Practice and Theory. The Pedagogy of Realistic Teacher Education (S. 32-50). New York: Routledge.

Krapp, A. (1999). Interest, motivation and learning: An educational-psychological perspective. European Journal of Psychology of Education, 14, 23-40.

Kuckartz, U. (2010). Einführung in die computergestützte Analyse qualitativer Daten. Wiesbaden: Springer.

Kuhn, D. (1991). The skills of argument. Cambridge: University Press.

Kuhn, D. (2009). Do students need to be taught how to reason? Educational Research Review, 4(1), 1-6.

Kuhn, D. (2015). Thinking together and alone. Educational Researcher, 44(1), 46-53.

Kuhn, D., Hemberger, L., \& Khait, V. (2016). Tracing the development of argumentive writing in a discourse-rich context. Written Communication, 33(1), 92-121. https://doi.org/10.1177/0741088315617157.

Kultusministerkonferenz (2012). Bildungsstandards im Fach Deutsch für Allgemeine Hochschulreife. Beschluss der Kultisministerkonferenz. https://www.kmk.org/fileadmin/veroeffentlichungen_ beschluesse/2012/2012_10_18-Bildungsstandards-Deutsch-Abi.pdf. Zugegriffen: 12. Juni 2017.

Kultusministerkonferenz (2014). Standards für die Lehrerbildung: Bildungswissenschaften. Beschluss der Kultusministerkonferenz. https://www.kmk.org/fileadmin/veroeffentlichungen_beschluesse/2004/ 2004_12_16-Standards-Lehrerbildung-Bildungswissenschaften.pdf. Zugegriffen: 30. Sept. 2017.

Lachner, A., Jarodzka, H., \& Nückles, M. (2016). What makes an expert teacher? Investigating teachers' professional vision and discourse abilities. Instructional Science, 44(3), 197-203.

Latifan, M., \& Bashash, L. (2004). Relation of value epistemological beliefs scale and judgments of two similar scenarios attributed to two different authorities. Psychological Reports, 95, 371-385.

Leinhardt, G., \& Greeno, J.G. (1986). The cognitive skill of teaching. Journal of Educational Psychology, $78(2), 75-95$

Leitão, S. (2000). The potential of argument in knowledge building. Human Development, 43, 332-360.

List, A., \& Alexander, P. A. (2017). Cognitive affective engagement model of multiple source use. Educational Psychologist, 52(3), 182-199.

Lunn Brownlee, J., Ferguson, L.E., \& Ryan, M. (2017). Changing teachers' epistemic cognition: a new conceptual framework for epistemic reflexivity. Educational Psychologist, 52(4), 242-252.

Maier, M., Rothmund, T., Retzbach, A., Otto, L., \& Besley, J.C. (2014). Informal learning through science media usage. Educational Psychologist, 49(2), 86-103.

Mattern, J., \& Bauer, J. (2014). Does teachers' cognitive self-regulation increase their occupational wellbeing? The structure and role of self-regulation in the teaching context. Teaching and Teacher Education, 43, 58-68.

Mercier, H., \& Sperber, D. (2011). Why do humans reason? Arguments for an argumentative theory. Behavioral and Brain Sciences, 34(2), 57-111.

Mertens, S., \& Gräsel, C. (2018). Entwicklungsbereiche bildungswissenschaftlicher Kompetenzen von Lehramtsstudierenden im Praxissemester. Zeitschrift für Erziehungswissenschaft, 21(1), 1-25.

Moshman, D., \& Tarricone, P. (2016). Logical and causal reasoning. In J. Greene, W. A. Sandoval \& I. Bråten (Hrsg.), Handbook of Epistemic Cognition (S. 54-67). New York: Routledge.

Muis, K. R., Bendixen, L. D., \& Haerle, F. C. (2006). Domain-generality and domain-specificity in personal epistemology research: Philosophical and empirical reflections in the development of a theoretical framework. Educational Psychology Review, 18(1), 3-54.

Neuweg, G. H. (2011). Das Wissen der Wissensvermittler. Problemstellungen, Befunde und Perspektiven der Forschung zum Lehrerwissen. In E. Terhart, H. Bennewitz \& M. Rothland (Hrsg.), Handbuch der Forschung zum Lehrerberuf (S. 583-614). Münster: Waxmann.

Neuweg, G. H. (2015). Kontextualisierte Kompetenzmessung. Eine Bilanz zu aktuellen Konzeptionen und forschungsmethodischen Zugängen. Zeitschrift für Pädagogik, 61(3), 377-383.

Newell, G. E., Beach, R., Smith, J., Vanderheide, J., Kuhn, D., \& Andriessen, J. (2011). Teaching and learning argumentative reading and writing: A review of research. Reading Research Quarterly, 46(3), 273-304.

Nückles, M., \& Schuba, C. (2019). „Teachers as Informed Pragmatists“: Ein theoretisches Modell und empirische Befunde zur Förderung didaktischer Argumentationskompetenz von angehenden Lehrkräften. In Profilbildung Lehramt - Konturen für Studium, Fächer, Universität. Ergebnisse des Kieler Programmworkshops der Qualitätsoffensive Lehrerbildung. Berlin: BMBF.

Nussbaum, E. M. (2008). Using argumentation vee diagrams (AVDs) for promoting argument-counterargument integration in reflective writing. Journal of Educational Psychology, 100(3), 549-565.

Osborne, J.F. (2010). Arguing to learn in science: The role of collaborative, critical discourse. Phi Delta Kappan, 328, 463-467. 
Osborne, J.F., \& Patterson, A. (2011). Scientific argument and explanation: A necessary distinction? Science Education, 95(4), 627-638.

Prenzel, M. (2010). Geheimnisvoller Transfer? Zeitschrift für Erziehungswissenschaft, 13(1), 21-37.

Renkl, A., Mandl, H., \& Gruber, H. (1996). Inert knowledge: Analysis and remedies. Educational Psych, $31(2), 115-121$.

Sackett, D.L., Rosenberg, W. M.C., Gray, J. A. M., Haynes, B. R., \& Richardson, S. W. (1996). Evidence based medicine: What it is and what it isn't. It's about integrating individual clinical expertise and the best external evidence. BMJ, 312(7023), 71-72.

Schellenbach-Zell, J., Fussangel, K., Erpenbach, A.L., \& Rochnia, M. (2018). Entwicklung eines Instruments zur Einschätzung der Reflexionskompetenz im Praxissemester. In I. Biederbeck \& M. Rothland (Hrsg.), Praxisphasen in der Lehrerbildung im Fokus der Bildungsforschung (S. 177-186). Münster: Waxmann.

Schön, D. A. (1983). The reflective practitioner. How professionals think in action. London: Routledge.

Schön, D. A., \& Argyris, C. (1996). Organizational learning II: Theory, method and practice. Asia Pacific Journal of Human Resources, 36, 107-109.

Shavelson, R.J. (2018). Role of scientific education research in professional action: A personal odyssey. Keynote auf der 6. Tagung der Gesellschaft für empirische Bildungsforschung (GEBF), Basel, 15.-17. Februar.

Shulman, L. S. (1987). Knowledge and teaching: Foundations of the new reform. Harvard Educational Review, 57(1), 1-23.

Sinatra, G. M. (2016). Thoughts on knowledge about thinking about knowledge. In J. A. Greene, W. A. Sandoval \& I. Bråten (Hrsg.), Handbook of epistemic cognition (S. 479-491). New York: Routledge.

Sinatra, G. M., Kienhues, D., \& Hofer, B.K. (2014). Addressing challenges to public understanding of science: Epistemic cognition, motivated reasoning, and conceptual change. Educational Psychologist, 49(2), 123-138.

Stark, R. (2005). Constructing arguments in educational discourses. In H. Gruber, C. Harteis, R. Mulder \& M. Rehrl (Hrsg.), Bridging individual, organizational, and cultural aspects of professional learning (S. 64-71). Regensburg: S. Roderer.

Stark, R. (2017). Probleme evidenzbasierter bzw. -orientierter pädagogischer Praxis. Zeitschrift für Pädagogische Psychologie, 31(2), 99-110.

Stark, R., Herzmann, P., \& Krause, U.-M. (2010). Effekte integrierter Lernumgebungen - Vergleich problembasierter und instruktionsorientierter Seminarkonzeptionen in der Lehrerbildung. Zeitschrift für Pädagogik, 56(4), 548-563.

Stürmer, K., Könings, K. D., \& Seidel, T. (2013). Declarative knowledge and professional vision in teacher education: Effect of courses in teaching and learning. British Journal of Educational Psychology, 83, 467-483.

Tenorth, H.-E. (2006). Professionalität im Lehrerberuf. Zeitschrift für Erziehungswissenschaft, 9(4), 580-597.

Toulmin, S.E. (2003). The uses of argument. Cambridge: Cambridge University Press.

Trempler, K., Hetmanek, A., Wecker, C., Kiesewetter, J., Wermelt, M., Fischer, M. R., Fischer, F., \& Gräsel, C. (2015). Nutzung von Evidenz im Bildungsbereich. Validierung eines Instruments zur Erfassung von Kompetenzen der Informationsauswahl und Bewertung von Studien. In S. Blömeke \& O. ZlatkinTroitschanskaia (Hrsg.) Kompetenzen von Studierenden (Zeitschrift für Pädagogik: 61. Beiheft, S. 144-166). Weinheim: Beltz.

Wagner, K., Klein, M., Klopp, E., \& Robin, S. (2014). Theoretisieren für die Praxis: Förderung anwendbaren pädagogischen Wissens anhand advokatorischer Fehler. Journal Für Die LehrerInnenbildung, $14(1), 65-74$.

Walton, D., \& Macagno, F. (2015). A classification system for argumentation schemes. Argument and Computation, 6(3), 219-245.

Wenglein, S., Bauer, J., Heininger, S., \& Prenzel, M. (2015). Kompetenz angehender Lehrkräfte zum Argumentieren mit Evidenz: Erhöht ein Training von Heuristiken die Argumentationsqualität? Unterrichtswissenschaft, 43(3), 209-224.

Wolff, C.E., Jarodzka, H., \& Van den Bogert, N. (2016). Teacher vision: Expert and novice teachers' perception of problematic classroom management scenes. Instructional Science, 44(3), 243-265.

Wubbels, T., \& Korthagen, F. A. (1990). The effects of a pre-service teacher education program for the preparation of reflective teachers. Journal of Education for Teaching, 16(1), 29-43.

Zohar, A., \& Nemet, F. (2002). Fostering students' knowledge and argumentation skills through dilemmas in human genetics. Journal of Research in Science Teaching, 39(1), 35-62. 


\section{Weiterführende Literatur}

Bråten, I., Ferguson, L.E., Anmarkrud, Ø., Strømsø, H. I. (2013). Prediction of learning and comprehension when adolescents read multiple texts: the roles of word-level processing, strategic approach, and reading motivation. Reading and Writing, 26(3), 321-348.

Korthagen, F., Wubbels, T. (2001). Learning from practice. In F. Korthagen, J. Kessels, B. Koster, B. Lagerwerf T.Wubbels (Hrsg.), Linking practice and theory: the pedagogy of realistic teacher education (S. 32-50). London: Routledge. 